\title{
A Phenomenological One-Dimensional Model Describing Stress-Induced Solid Phase Transformation with Permanent Inelasticity
}

\author{
F. Auricchio \\ Dipartimento di Meccanica Strutturale, Università degli Studi di Pavia, Italy; \\ European School for Advanced Studies in Reduction of Seismic Risk (ROSE School), Università degli \\ Studi di Pavia, Italy and \\ Istituto di Matematica Applicata e Tecnologie Informatiche del CNR, Pavia, Italy
}

\author{
A. Reali \\ Dipartimento di Meccanica Strutturale, Universitá degli Studi di Pavia, Italy
}

The use of shape memory alloys for a large number of applications in many fields of engineering has given rise to a great interest of researchers on an exhaustive modeling of their macroscopic behaviour.

Most of the models proposed in the literature, however, consider a perfect pseudo-elastic behaviour (i.e. no residual strains), which is experimentally proved to be only an approximation. This paper addresses a new one-dimensional model able to include also permanent inelasticity effects, whose main features are shown by many numerical tests.

\section{INTRODUCTION}

In last years, the research on the constitutive modeling of shape memory alloys (SMA) has greatly increased, due to the large number of SMA industrial applications in many branches of engineering $[1,2]$. Accordingly, many models able to reproduce one or both of the SMA macroscopic behaviours (i.e. pseudo-elasticity and shape-memory effect) have been recently proposed in the literature (see e.g. [3-12]). Among them, the one proposed in [13] and improved in [14] seems to be very attractive for its potential. However, as in many other models present in the literature, residual inelastic strain effects are not included, while experimental results as the ones shown in Figure 1 (cf. [15]) prove them not to be negligible. Moreover, Figure 1 also highlights the need for the introduction of degradation effects.

It is in this framework that the present work takes its justification; in fact, we wish to propose a new phenomenological one-dimensional constitutive model, able to include permanent

Received 26 April 2006.

Address correspondence to A. Reali, Dipartimento di Meccanica Strutturale, Università degli Studi di Pavia, Via Ferrata 1, 27100, Pavia Italy. E-mail: alessandro.reali@unipv.it inelasticity and degradation effects together with classical SMA macroscopic behaviours. In the following, an analytical description of the constitutive equations is presented and many numerical experiments are performed in order to show the features of the model.

\section{1D MODEL FOR STRESS-INDUCED SOLID PHASE TRANSFORMATION INCLUDING PERMANENT INELASTICITY}

Starting from the SMA constitutive model discussed in [14] and limiting the development to a 1D situation, we describe now an extended set of constitutive equations, including a new internal variable able to activate and take into account the effect of residual inelastic strains.

\subsection{Time-Continuous Frame}

The total strain $\varepsilon$ is assumed as control variable, the transformation strain $\varepsilon^{t r}$ and the residual inelastic strain $q$ as internal ones. The quantity $\varepsilon^{t r}$ plays the role of describing the strain associated to the phase transformation as in [14], but here it should not be a fully reversible quantity, i.e., as the stress is driven to zero, the transformation strain should not be completely recovered, but it should tend to the value of the residual inelastic strain $q$. Moreover, the following condition is required:

$$
\left|\varepsilon^{t r}\right| \leq \varepsilon_{L}
$$

where $\varepsilon_{L}$ is a material parameter corresponding to the maximum transformation strain reached at the end of the transformation.

Assuming a small strain regime, the free energy function $\Psi$ for a polycrystalline SMA material is expressed as the convex 


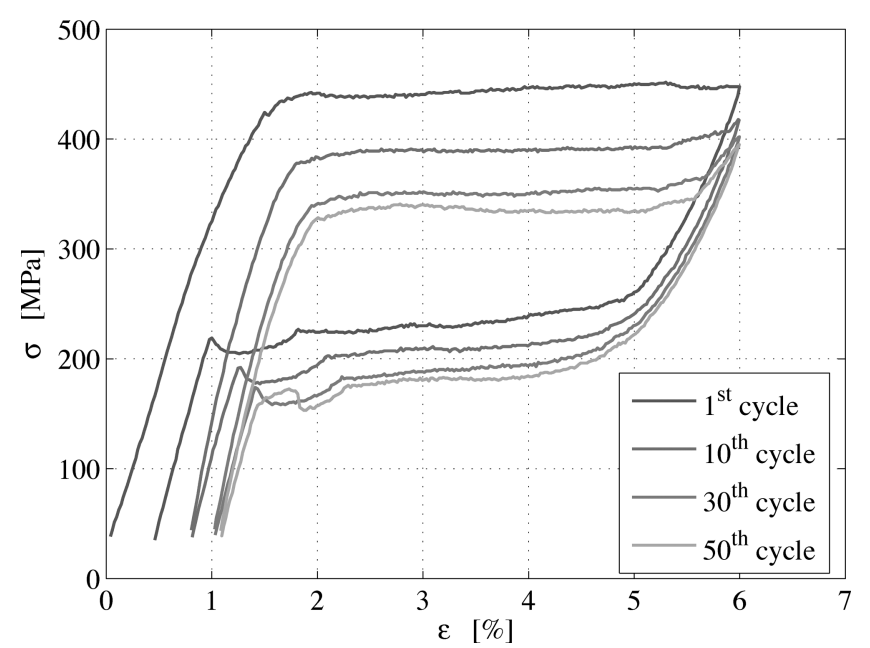

FIG. 1. Experimental results on a SMA wire. Cyclic tension test: stress versus strain up to $6 \%$ strain.

potential:

$$
\begin{aligned}
\Psi\left(\varepsilon, \varepsilon^{t r}, q\right) & =\frac{1}{2} E\left(\varepsilon-\varepsilon^{t r}\right)^{2}+\beta\left\langle T-M_{f}\right\rangle\left|\varepsilon^{t r}-q\right| \\
& +\frac{1}{2} h\left(\varepsilon^{t r}\right)^{2}+\frac{1}{2} H q^{2}-A \varepsilon^{t r} q+\mathcal{J}_{\mathcal{E}_{L}}\left(\varepsilon^{t r}\right),
\end{aligned}
$$

where $E$ is the Young's modulus, $\beta$ is a material parameter related to the dependence of the critical stress on the temperature, $T$ is the absolute temperature, $M_{f}$ is the temperature below which only martensite phase is stable, $h, H$ and $A$ are material parameters controlling, respectively, the slope of the linear stress-transformation strain relation, the saturation of the residual inelastic strain evolution and the bilinear coupling between $\varepsilon^{t r}$ and $q$. In (2) we make use of the indicator function $\mathcal{J}_{\varepsilon_{L}}\left(\varepsilon^{t r}\right)$ :

$$
\mathcal{J}_{\varepsilon_{L}}\left(\varepsilon^{t r}\right)=\left\{\begin{array}{cl}
0 & \text { if }\left|\varepsilon^{t r}\right|<\varepsilon_{L} \\
+\infty & \text { otherwise }
\end{array}\right.
$$

to satisfy the transformation strain constraint (1); moreover, we introduce the positive $\operatorname{part}\langle\bullet\rangle$ function:

$$
\langle a\rangle= \begin{cases}a & \text { if } a>0 \\ 0 & \text { otherwise. }\end{cases}
$$

Following standard arguments, we can derive from the free energy function $\Psi$ the constitutive equations:

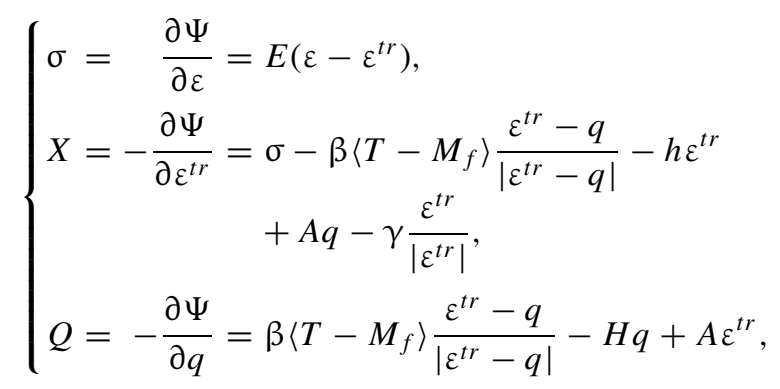

where $\sigma$ is the stress, while $X$ and $Q$ are thermodynamic stress-like quantities associated respectively to the transformation strain $\varepsilon^{t r}$ and to the residual inelastic strain $q$. The variable $\gamma$ is the result of the subdifferential of the indicator function and is defined as:

$$
\begin{cases}\gamma=0 & \text { if }\left|\varepsilon^{t r}\right|<\varepsilon_{L} \\ \gamma \geq 0 & \text { if }\left|\varepsilon^{t r}\right|=\varepsilon_{L} .\end{cases}
$$

We now introduce a limit function $F$ defined as:

$$
F(X, Q)=|X|+\mathrm{k}|Q|-R,
$$

where $\mathrm{K}$ is a scaling modulus for the inelastic effect and $R$ is a material parameter defining the radius of the elastic domain and controlling the width of the hysteresis loops (cf. [14]). Considering an associative framework, the flow rules for internal variables take the form:

$$
\left\{\begin{array}{l}
\dot{\varepsilon}^{t r}=\dot{\zeta} \frac{\partial F}{\partial X}=\dot{\zeta} \frac{X}{|X|}, \\
\dot{q}=\dot{\zeta} \frac{\partial F}{\partial Q}=\dot{\zeta} \kappa \frac{Q}{|Q|} .
\end{array}\right.
$$

We finally complete the model by the classical Kuhn-Tucker conditions:

$$
\left\{\begin{array}{r}
\dot{\zeta} \geq 0 \\
F \leq 0 \\
\dot{\zeta} F=0
\end{array}\right.
$$

\section{Remark 1.}

The choice of the parameter $k$ controls the entity of the inelastic effect and it is not completely free. For simplicity, we show how to compute an upper bound for this parameter when $h=H=A=0 \mathrm{MPa}$ and $\left|\varepsilon^{t r}\right|<\varepsilon_{L}$. In this case the constitutive equations for $X$ and $Q$ take the form:

$$
\left\{\begin{array}{l}
X=\sigma-\beta\left\langle T-M_{f}\right\rangle \frac{\varepsilon^{t r}-q}{\left|\mathcal{E}^{t r}-q\right|}, \\
Q=\beta\left\langle T-M_{f}\right\rangle \frac{\varepsilon^{t r}-q}{\left|\mathcal{E}^{t r}-q\right|}
\end{array}\right.
$$

substituting in the expression (4) for the limit function and taking into account the second Kuhn-Tucker condition $(6)_{2}$, we get:

$$
F=|X|+\kappa \beta\left\langle T-M_{f}\right\rangle-R \leq 0 .
$$

In order to satisfy this inequality, since $|X| \geq 0$, it is necessary to have:

$$
\kappa \beta\left\langle T-M_{f}\right\rangle-R \leq 0,
$$

that leads to the following upper bound for the parameter $\mathrm{K}$ :

$$
\kappa \leq \frac{R}{\beta\left\langle T-M_{f}\right\rangle} .
$$


We finally highlight that the choice $\mathrm{K}=0$ leads to recover the model without permanent inelasticity discussed in [14]. In fact, setting $\mathrm{K}=0$ in (5) $)_{2}$, we get $\dot{q}=0$, which means that $q$ does not evolve and is always equal to its initial value, i.e. $q \equiv 0$.

\section{Remark 2.}

In many experimental studies it has been observed that SMA materials show a not indefinitely evolving inelasticity, that saturates after reaching a certain level. In our model we can introduce such an effect by choosing a non-zero $H$ parameter. We start noting that the evolution of $q$ necessarily stops when $Q=0$. Then, considering for simplicity the case $A=0 \mathrm{MPa}$, for this condition we can obtain a priori the saturated value of $q$ from (3) 3 , i.e.:

$$
q_{\max }^{ \pm}= \pm \frac{\beta\left\langle T-M_{f}\right\rangle}{H},
$$

which is dependent only on the temperature.

On the other hand, for $A \neq 0 \mathrm{MPa}$ the condition $Q=0$ leads to a relation from which it is not possible to obtain a simple a priori expression for $q_{\mathrm{max}}^{ \pm}$, due to the coupling between $q$ and $\varepsilon^{t r}$. In such a case, the value of $q_{\max }^{ \pm}$will not be dependent only on the temperature.

Moreover, even if sometimes experimental results show that the loading rate can influence the maximum level of permanent inelastic strain, we stress here that such dependence is not reproduced by the present model.

We finally remark that for $T<M_{f}$ (i.e. in the case of stable martensite phase) an evolution for $q$ is obtained only for a nonzero value of $A$.

\subsection{Time-Discrete Frame}

We now introduce a subdivision of the time interval of interest $\left[0, t_{f}\right]$ and solve the evolution problem over the generic interval $\left[t_{n}, t_{n+1}\right]$ with $t_{n+1}>t_{n}$. Note that in the following, for the sake of notation simplicity, we drop the subindex $n+1$ for all the variables computed at time $t=t_{n+1}$. We remark that we assume the temperature to be assigned at each time step in order to treat only the mechanical part of the problem.

\subsubsection{Time Integration}

Supposed known the solution at time $t_{n}$ and the value of the total strain at time $t_{n+1}$, an implicit backward Euler scheme is employed to integrate the flow rules for the internal variables. In order to differentiate in zero the free energy $\Psi$ and the limit function $F$, we substitute the terms $\left|\varepsilon^{t r}-q\right|$ and $|Q|$ with $\overline{\left|\mathcal{E}^{t r}-q\right|}$ and $\overline{|Q|}$, where $\overline{|\bullet|}$ is a regularized absolute value defined as:

$$
\overline{|a|}=\sqrt{|a|^{2}+\delta}-\sqrt{\delta}
$$

with $\delta$ user-defined parameter controlling the regularization smoothness. We stress that, as we introduce a regularization only to obtain always differentiable functions and to guarantee the numerical robustness of the model, the parameter $\delta$ can be chosen to be even very small (e.g., $10^{-8}$ ).
Hence, the time-discrete constitutive model can be written as:

$$
\left\{\begin{aligned}
\sigma= & E\left(\varepsilon-\varepsilon^{t r}\right), \\
X= & \sigma-\beta\left\langle T-M_{f}\right\rangle \frac{\varepsilon^{t r}-q}{\sqrt{\left|\varepsilon^{t r}-q\right|^{2}+\delta}} \\
& -h \varepsilon^{t r}+A q-\gamma \frac{\varepsilon^{t r}}{\mid \varepsilon^{t r \mid}}, \\
Q= & \beta\left\langle T-M_{f}\right\rangle \frac{\varepsilon^{t r}-q}{\sqrt{\left|\varepsilon^{t r}-q\right|^{2}+\delta}}-H q+A \varepsilon^{t r}, \\
\mathcal{E}^{t r}= & \varepsilon_{n}^{t r}+\Delta \zeta \frac{X}{|X|}, \\
q= & q_{n}+\Delta \zeta \kappa \frac{Q}{\sqrt{|Q|^{2}+\delta}}, \\
F= & |X|+\kappa|Q| Q \mid-R,
\end{aligned}\right.
$$

with the requirements:

$$
\left\{\begin{array}{l}
\gamma \quad \geq 0 \\
\left|\varepsilon^{t r}\right| \leq \varepsilon_{L}, \\
\Delta \zeta \geq 0, \quad F \leq 0, \quad \Delta \zeta F=0
\end{array}\right.
$$

where $\Delta \zeta=\zeta-\zeta_{n}=\int_{t_{n}}^{t_{n+1}} \dot{\zeta} \mathrm{d} t$ is the time-integrated consistency parameter.

\subsubsection{Solution}

The solution of the discrete model is performed by means of an elastic-predictor inelastic-corrector return map procedure as in classical plasticity problems [16]. An elastic trial state is evaluated for frozen internal variables and a trial value of the limit function is computed in order to verify the admissibility of the trial state; if this is not verified, the step is inelastic and the evolution equations are integrated.

As in [14], we distinguish two inelastic phases in our model: a non-saturated phase $\left(\left|\varepsilon^{t r}\right|<\varepsilon_{L}, \gamma=0\right)$ and a saturated one $\left(\left|\varepsilon^{t r}\right|=\varepsilon_{L}, \gamma \geq 0\right)$. In our solution procedure we assume to be in a non-saturated phase, and at convergence we check if our assumption is violated. If the non-saturated solution is not admissible, then we search for a new solution under saturated conditions.

At each inelastic step, the nonlinear system constituted by equations (7) has to be solved. As the aim of this paper is showing the model behaviour (and not focusing on algorithmic problems), we find a solution to the system using the function fsolve implemented in the optimization toolbox of the program MATLAB ${ }^{\circledR}$.

\section{NUMERICAL RESULTS}

In this Section we perform a number of stress-driven tests in order to show the model behaviour when reproducing main macroscopic SMA features. Unless otherwise stated, in all the performed tests we consider the material properties specified in Table 1. 
TABLE 1

Material parameters

\begin{tabular}{lrc}
\hline Parameter & Value & Unit \\
\hline$E$ & $5 \cdot 10^{4}$ & $\mathrm{MPa}$ \\
$\beta\left\langle T-M_{f}\right\rangle$ & 150 & $\mathrm{MPa}$ \\
$h$ & 1000 & $\mathrm{MPa}$ \\
$R$ & 50 & $\mathrm{MPa}$ \\
$\varepsilon_{L}$ & 4 & $\%$ \\
$\delta$ & $10^{-8}$ & - \\
\hline
\end{tabular}

The aim of the following numerical experiments is to investigate the different effects controlled by each model parameter. For every test, we plot the stress input history, the output stressstrain curve and the output histories for the internal variables $\left(\varepsilon^{t r}\right.$ and $q$ ) and the associated stress-like quantities ( $X$ and $Q$ ).

- Test 1: tension cycles with $H=0 \mathrm{MPa}, A=0 \mathrm{MPa}$ and $\mathrm{k}=2 \%$.

The first performed numerical experiment consists of tension cycles reaching a maximum stress of $\sigma_{\max }=$ $300 \mathrm{MPa}$. The choice of a non-zero parameter $\mathrm{k}$ gives rise to a permanent inelasticity phenomenon. Figures 2 and 3 refer to a single tension cycle, while Figures 4 and 5 refer to ten tension cycles. A significant evolution of $q$, which represents the level of transformation strain that is not recovered during the unloading phase to $\sigma=$ 0 , is observed.

- Test 2: ten tension cycles with $H=1.5 \cdot 10^{4} \mathrm{MPa}$, $A=0 \mathrm{MPa}$ and $\mathrm{k}=2 \%$.

In this test we verify what is highlighted by the second Remark of Section 2. The second plot in Figure 7 shows that the internal variable $q$ does not exceed the threshold $\beta\left\langle T-M_{f}\right\rangle / H=150 /\left(1.5 \cdot 10^{4}\right)=1 \%$, which is also the value of residual strain shown by the stable stress-strain cycles of Figure 6.

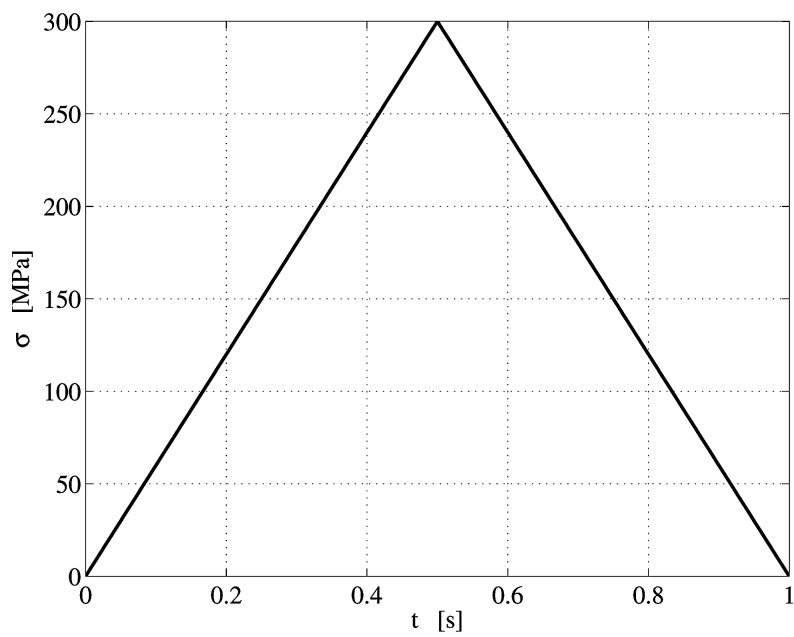

- Test 3: ten tension cycles with $H=1.5 \cdot 10^{4} \mathrm{MPa}$, $A=0 \mathrm{MPa}$ and $\mathrm{K}=4 \%$.

This numerical experiment is similar to the previous one, but here the parameter $\mathrm{K}$ is taken equal to the double as before, implying a greater inelastic effect, reaching its maximum value $(q=1 \%)$ in half the time as before (see Figures 8 and 9).

- Test 4: tension and compression cycles with $H=1.5$. $10^{4} \mathrm{MPa}, A=0 \mathrm{MPa}$ and $\mathrm{\kappa}=2 \%$.

In order to properly assess the behaviour of this model it is convenient to test it under cycles both in tension and compression. In Figures 10 and 11 we report the results of a loading history constituted by ten tension cycles followed by a compression one, while in Figures 12 and 13 the results of a loading history of ten tension and ten compression cycles; the good performance of the model are confirmed by both of these tests.

- Test 5: twenty tension cycles with $H=1.5 \cdot 10^{4} \mathrm{MPa}$, $A=10^{3} \mathrm{MPa}$ and $\mathrm{\kappa}=2 \%$.

This experiment is aimed at investigating the effect on the model of the parameter $A$ coupling the two internal variables. As shown by Figure 14 (right), a value of $A$ different from zero results in shifting down the loops. This sort of degradation effect is an important feature of the model as an analogous phenomenon is observed in experimental tests, as proved by Figure 1.

- Test 6: tension cycle with $T<M_{f}$ and strain recovery by heating; $H=0 \mathrm{MPa}, A=10^{3} \mathrm{MPa}$ and $\mathrm{K}=2 \%$.

The goal of this last test is to study the behaviour of the model when reproducing the shape-memory effect. The input consists in a tension cycle with a maximum stress $\sigma_{\max }=100 \mathrm{MPa}$ at a temperature $T<M_{f}$ (which implies $\beta\left\langle T-M_{f}\right\rangle=0 \mathrm{MPa}$ ), followed by a heating process at a constant zero stress up to a temperature such that $\beta\left\langle T-M_{f}\right\rangle=100 \mathrm{MPa}$. Figures 16 and 17 show that even in this case a inelastic effect is activated, so that we observe only a partial shape-recovery.

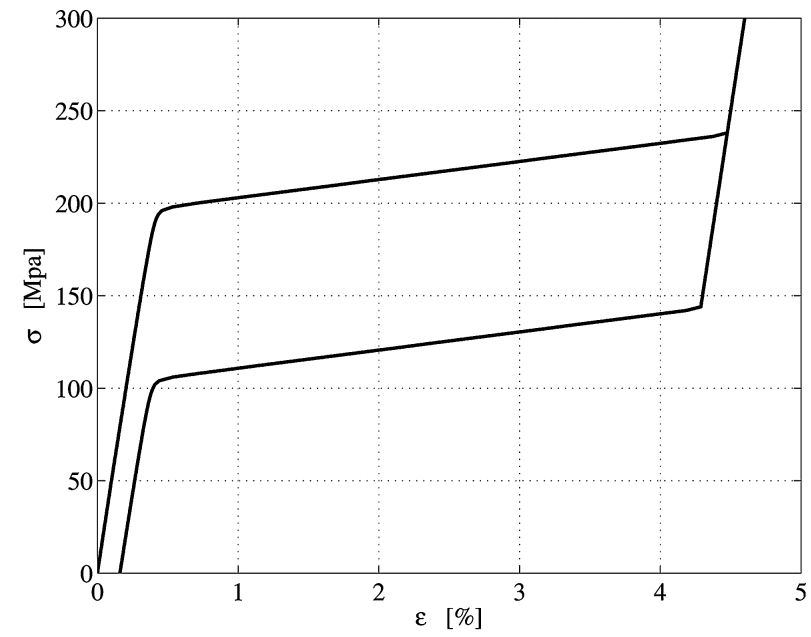

FIG. 2. Test 1 : single tension cycle with $H=0 \mathrm{MPa}, A=0 \mathrm{MPa}$ and $\mathrm{k}=2 \%$. Input stress history and stress-strain output. 

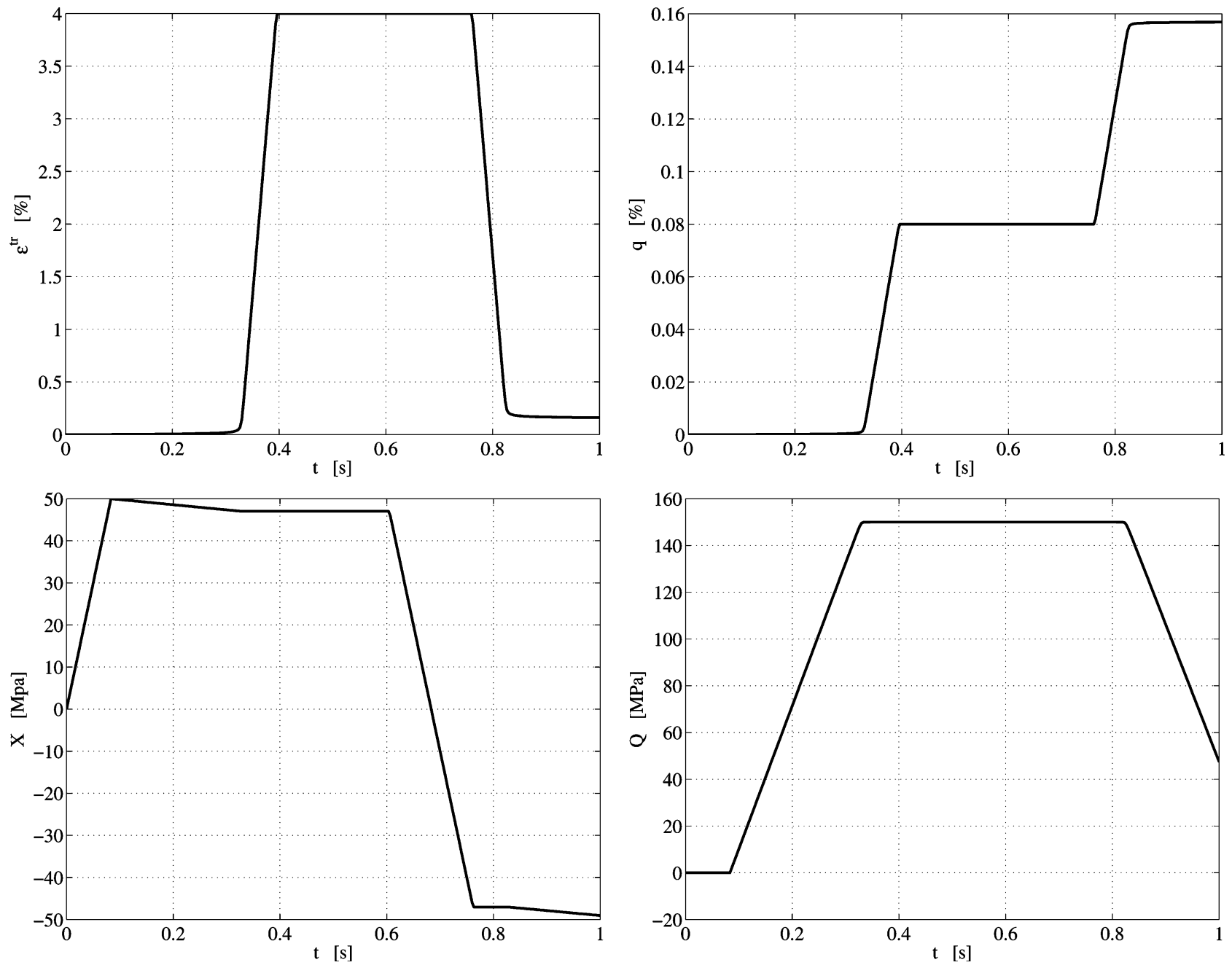

FIG. 3. Test 1: single tension cycle with $H=0 \mathrm{MPa}, A=0 \mathrm{MPa}$ and $\mathrm{\kappa}=2 \%$. Output histories for $\varepsilon^{t r}, q, X$ and $Q$.
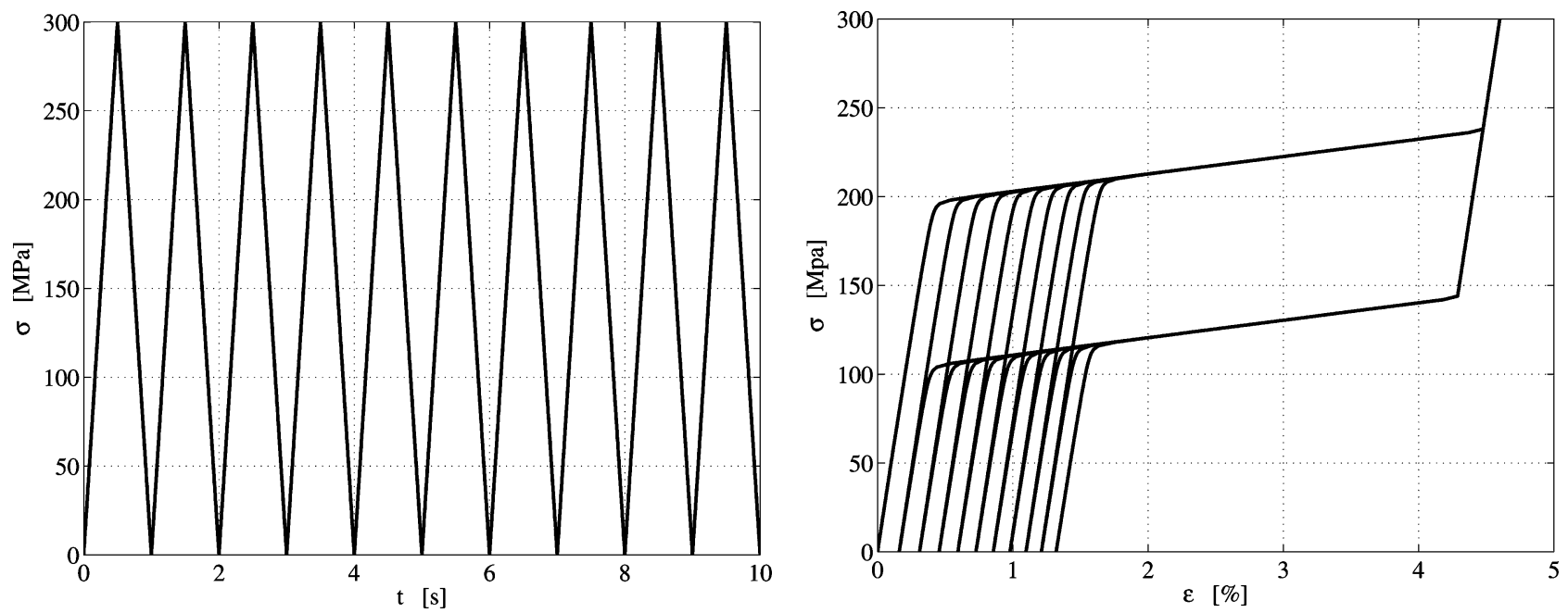

FIG. 4. Test 1: ten tension cycles with $H=0 \mathrm{MPa}, A=0 \mathrm{MPa}$ and $\mathrm{k}=2 \%$. Input stress history and stress-strain output. 

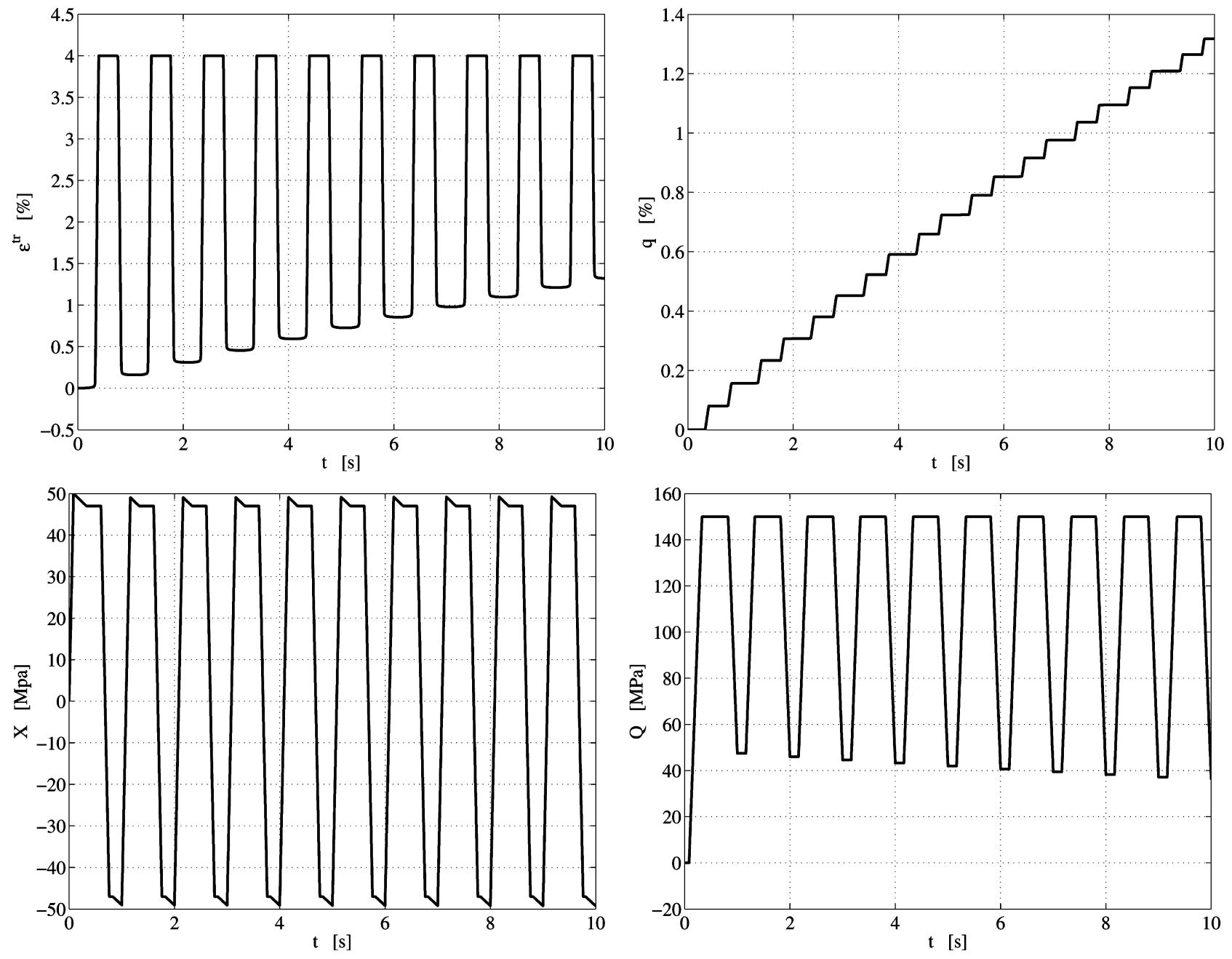

FIG. 5. Test 1: ten tension cycles with $H=0 \mathrm{MPa}, A=0 \mathrm{MPa}$ and $\mathrm{\kappa}=2 \%$. Output histories for $\varepsilon^{t r}, q, X$ and $Q$.
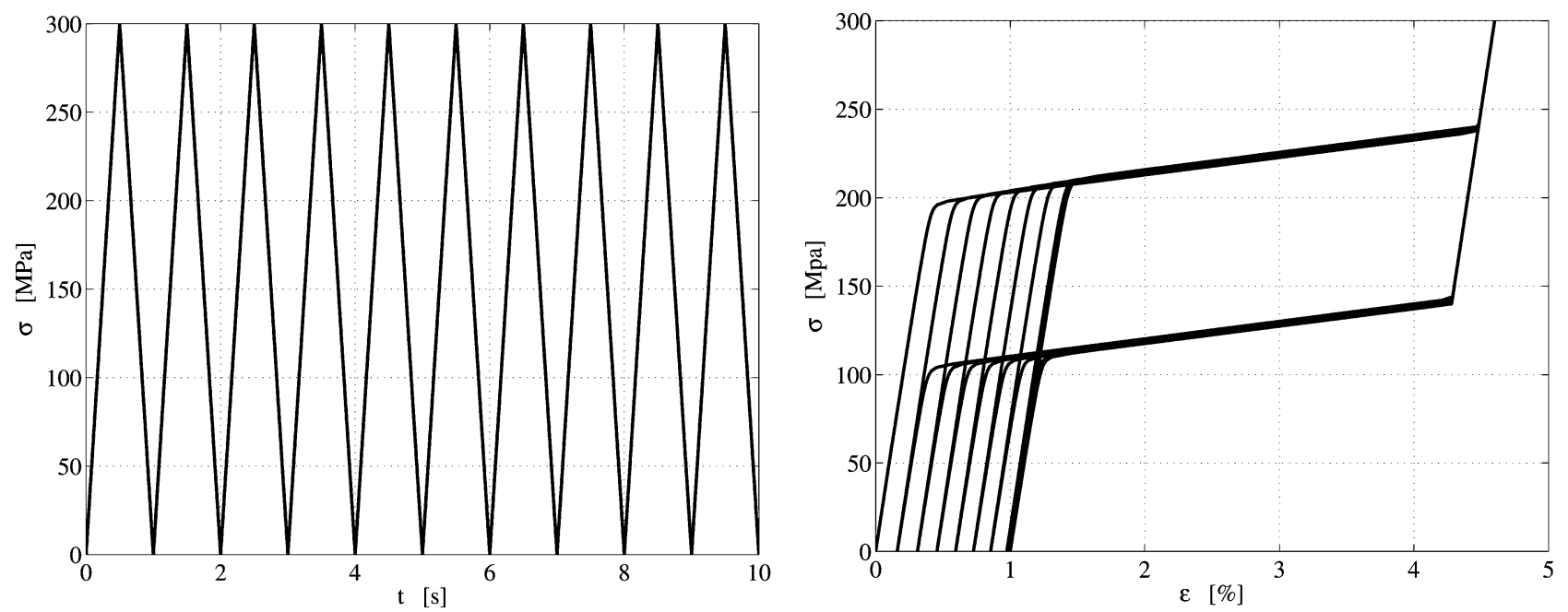

FIG. 6. Test 2: ten tension cycles with $H=1.5 \cdot 10^{4} \mathrm{MPa}, A=0 \mathrm{MPa}$ and $\mathrm{k}=2 \%$. Input stress history and stress-strain output. 

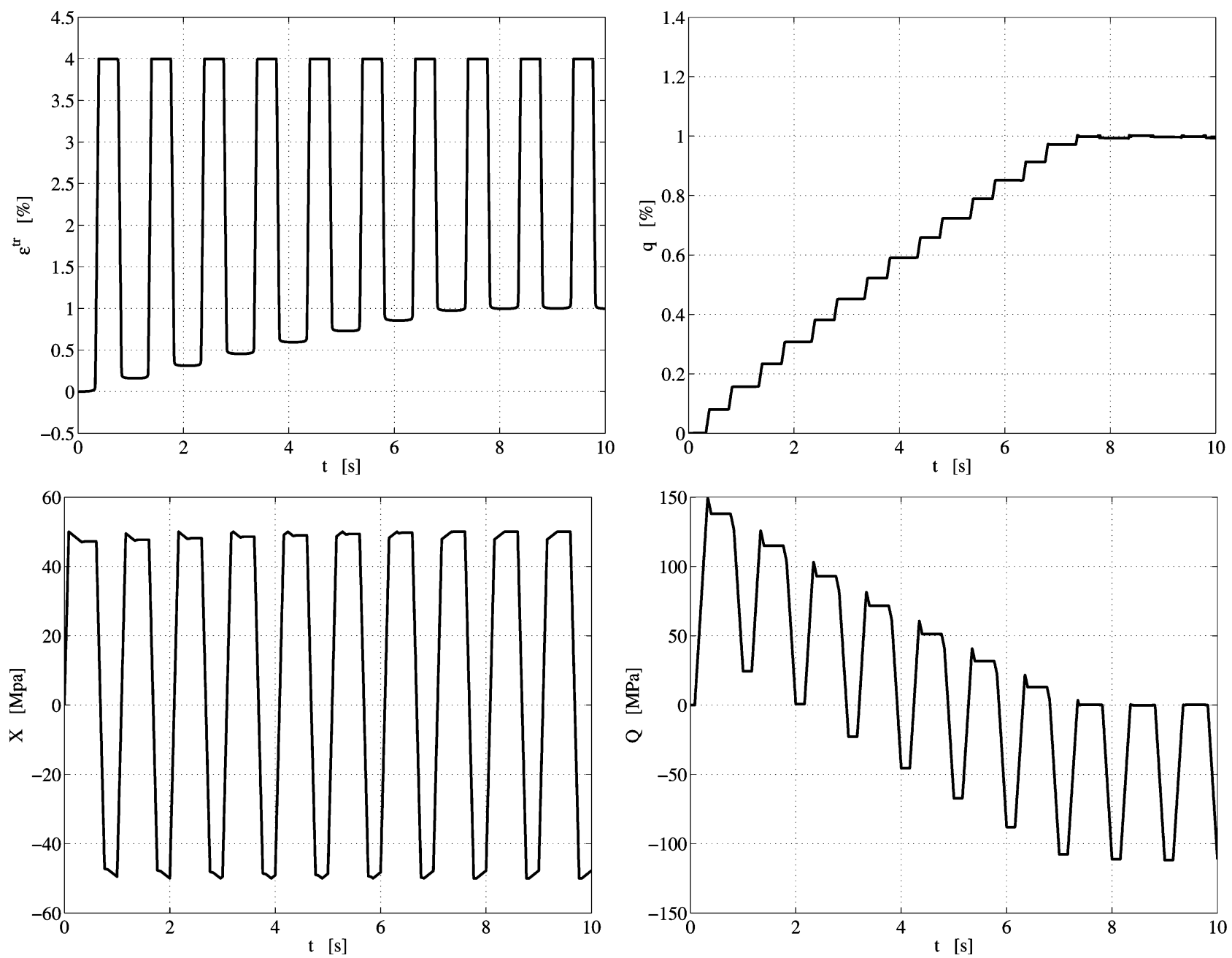

FIG. 7. Test 2: ten tension cycles with $H=1.5 \cdot 10^{4} \mathrm{MPa}, A=0 \mathrm{MPa}$ and $\mathrm{\kappa}=2 \%$. Output histories for $\varepsilon^{t r}, q, X$ and $Q$.
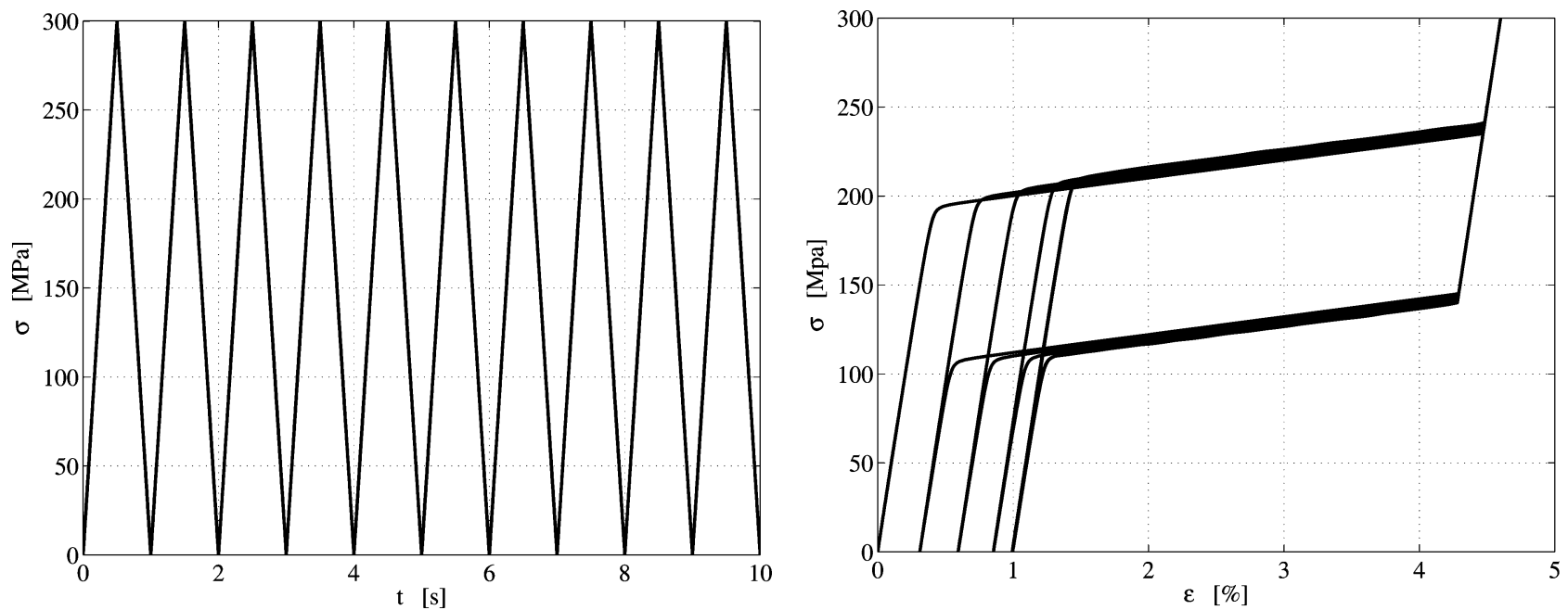

FIG. 8. Test 3: ten tension cycles with $H=1.5 \cdot 10^{4} \mathrm{MPa}, A=0 \mathrm{MPa}$ and $\mathrm{k}=4 \%$. Input stress history and stress-strain output. 

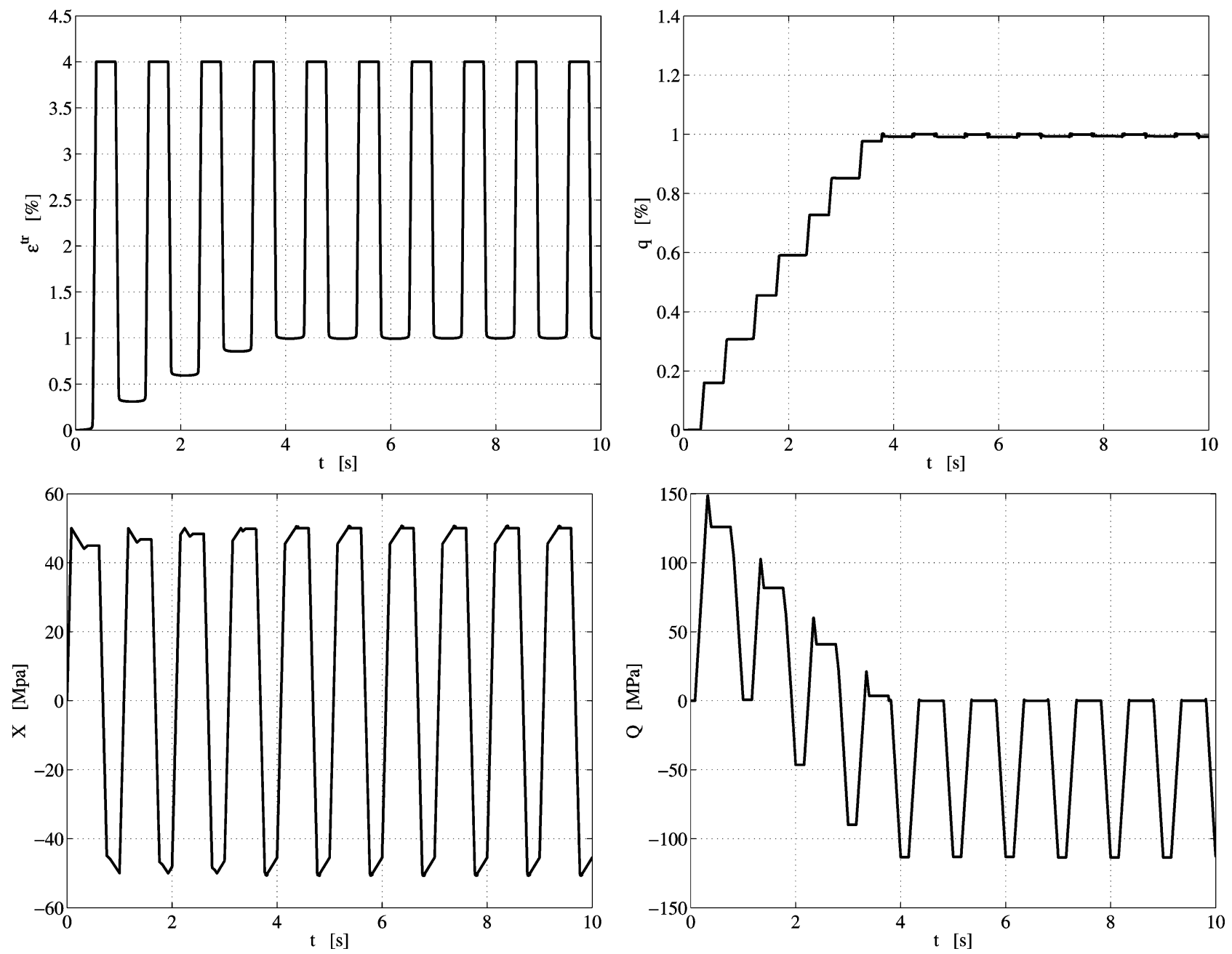

FIG. 9. Test 3: ten tension cycles with $H=1.5 \cdot 10^{4} \mathrm{MPa}, A=0 \mathrm{MPa}$ and $\mathrm{\kappa}=4 \%$. Output histories for $\varepsilon^{t r}, q, X$ and $Q$.
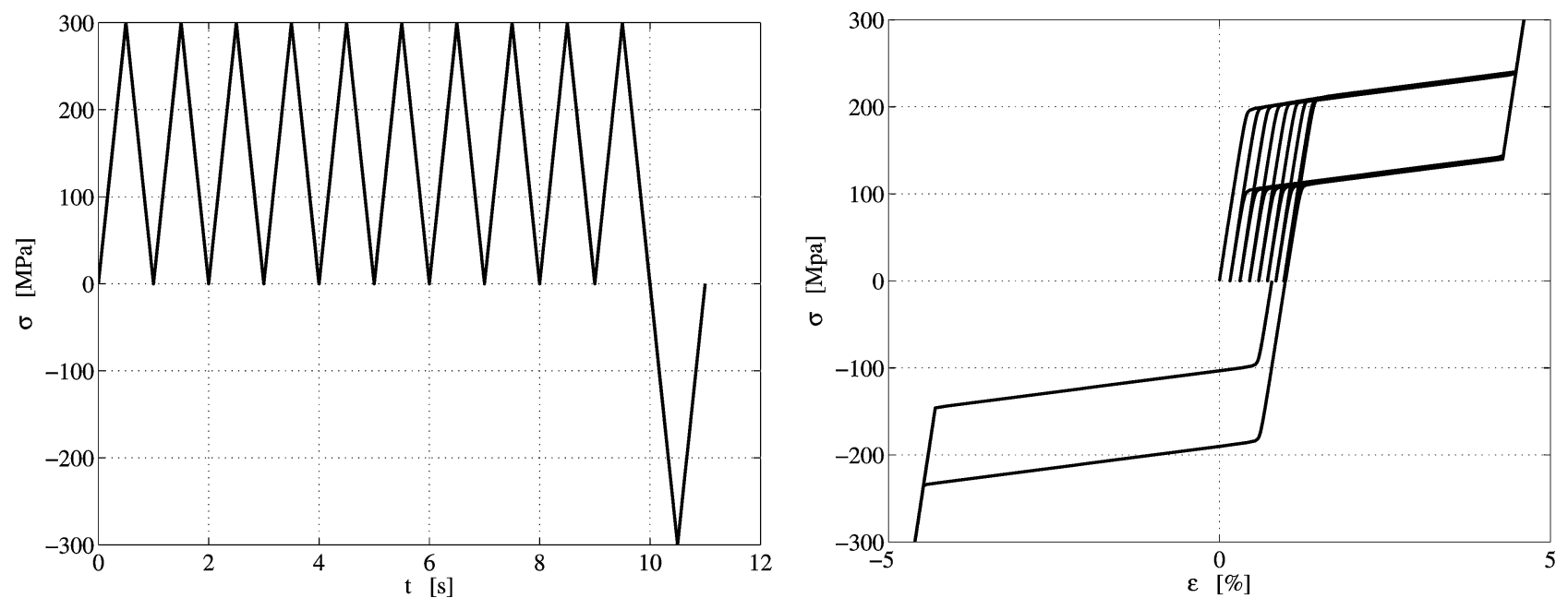

FIG. 10. Test 4: ten tension and one compression cycles with $H=1.5 \cdot 10^{4} \mathrm{MPa}, A=0 \mathrm{MPa}$ and $\mathrm{\kappa}=2 \%$. Input stress history and stress-strain output. 

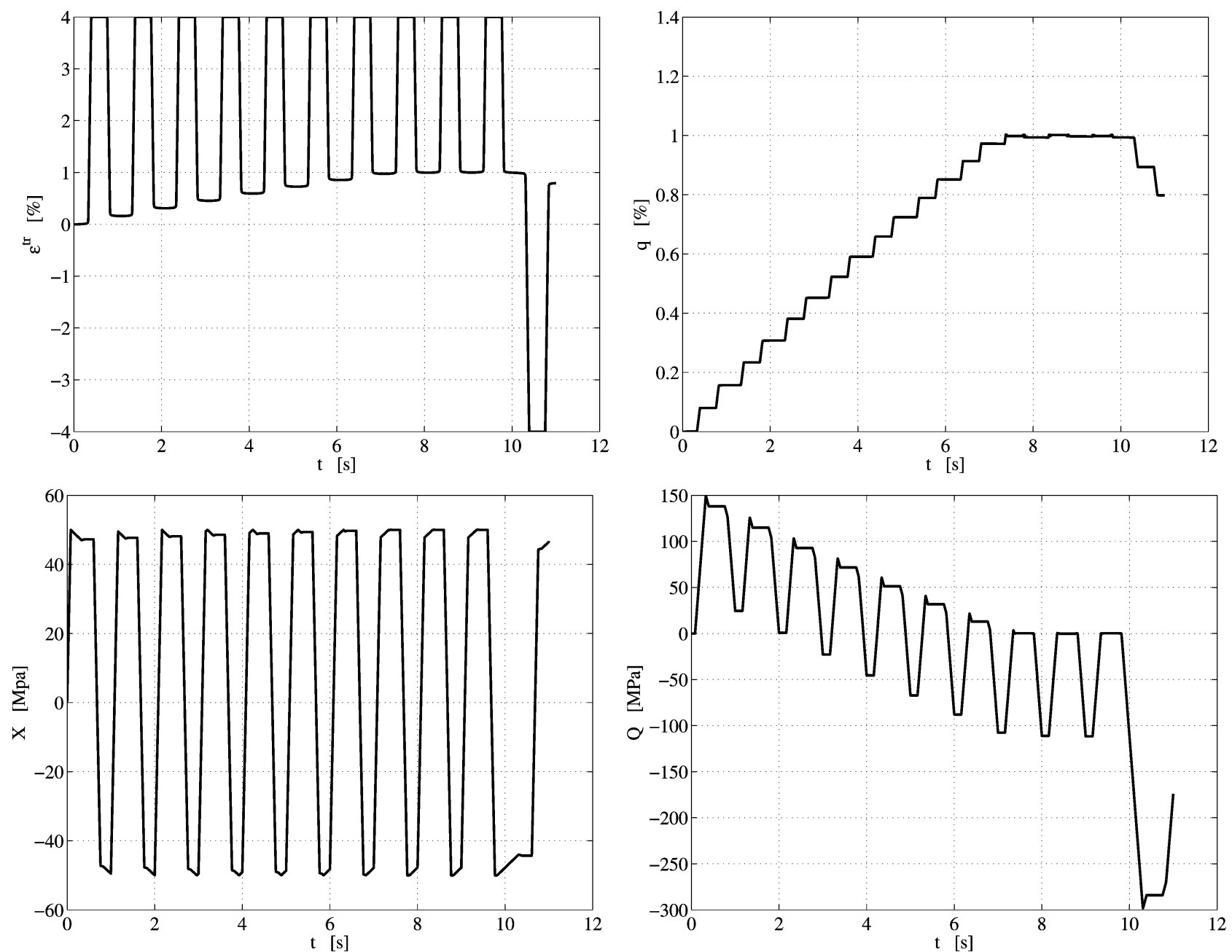

FIG. 11. Test 4: ten tension and one compression cycles with $H=1.5 \cdot 10^{4} \mathrm{MPa}, A=0 \mathrm{MPa}$ and $\mathrm{\kappa}=2 \%$. Output histories for $\varepsilon^{t r}, q$, $X$ and $Q$.
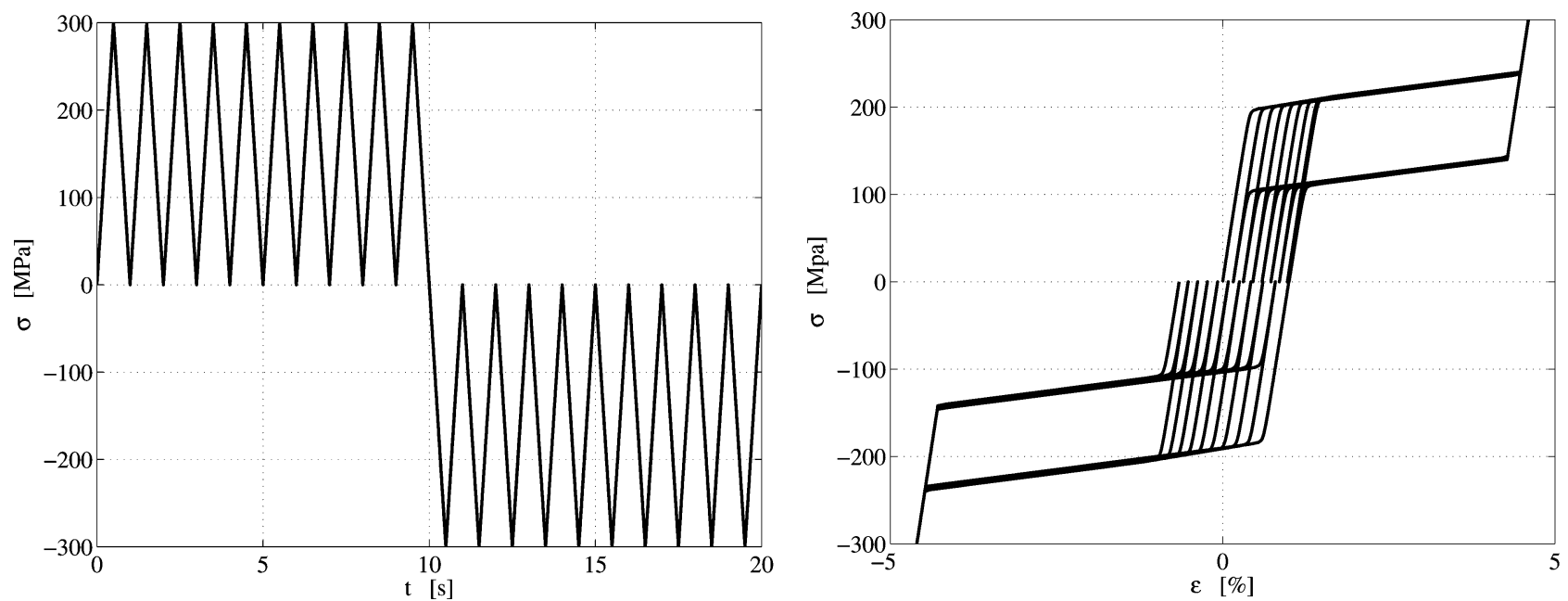

FIG. 12. Test 4: ten tension and ten compression cycles with $H=1.5 \cdot 10^{4} \mathrm{MPa}, A=0 \mathrm{MPa}$ and $\mathrm{k}=2 \%$. Input stress history and stress-strain output. 

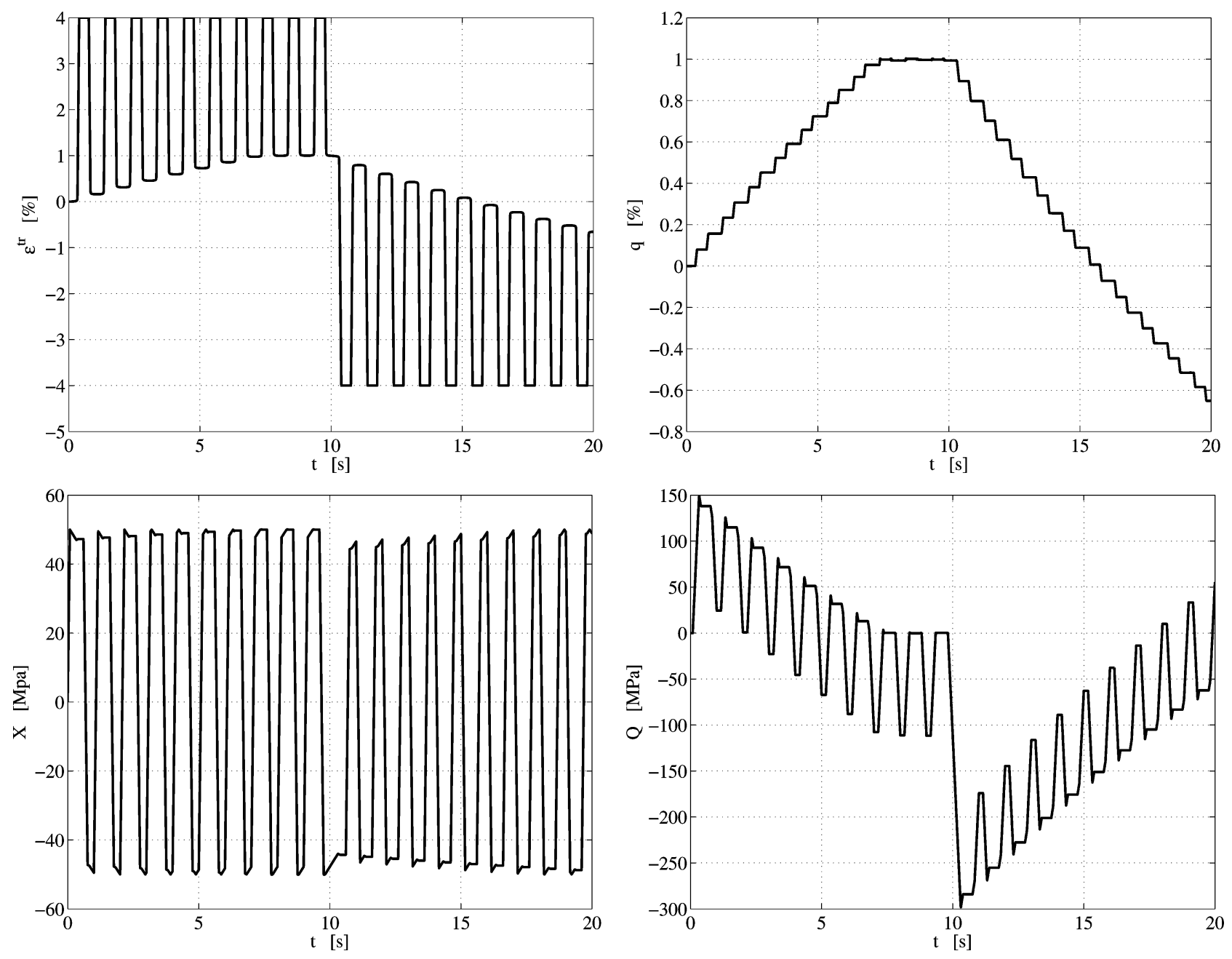

FIG. 13. Test 4: ten tension and ten compression cycles with $H=1.5 \cdot 10^{4} \mathrm{MPa}, A=0 \mathrm{MPa}$ and $\mathrm{\kappa}=2 \%$. Output histories for $\varepsilon^{t r}, q, X$ and $Q$.
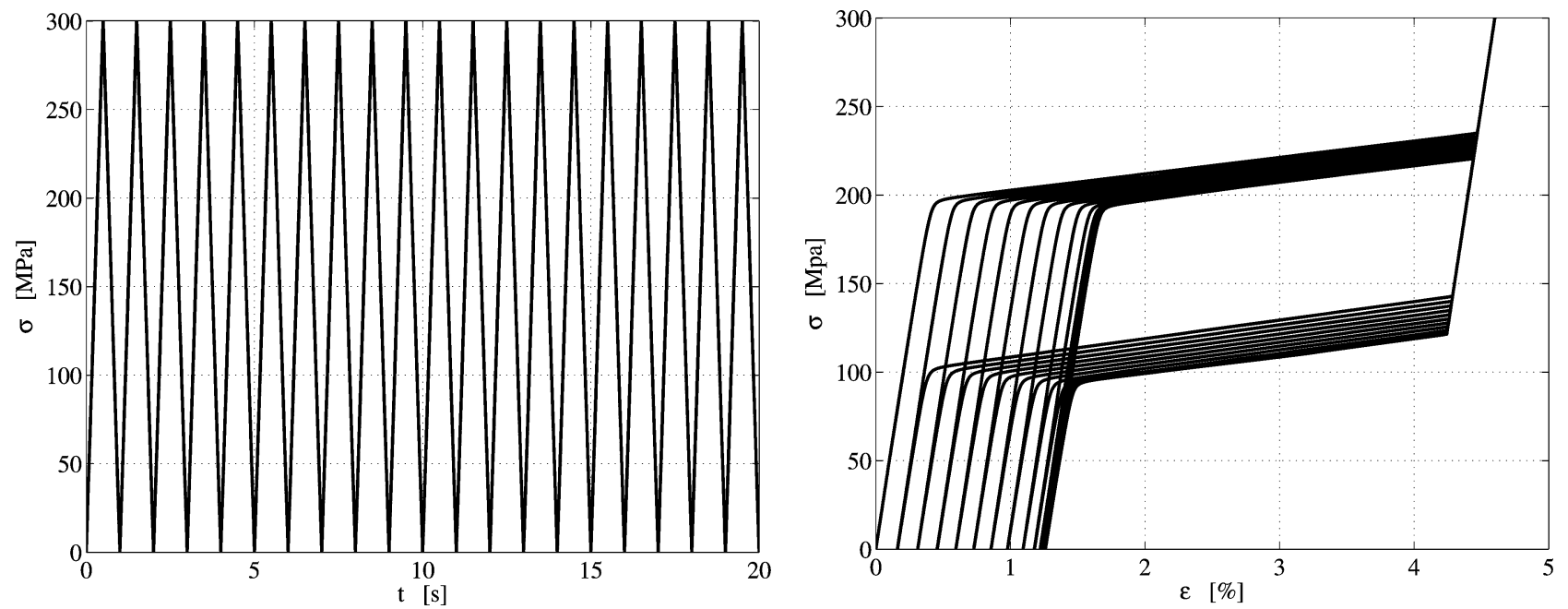

FIG. 14. Test 5: twenty tension cycles with $H=1.5 \cdot 10^{4} \mathrm{MPa}, A=10^{3} \mathrm{MPa}$ and $\mathrm{k}=2 \%$. Input stress history and stress-strain output. 

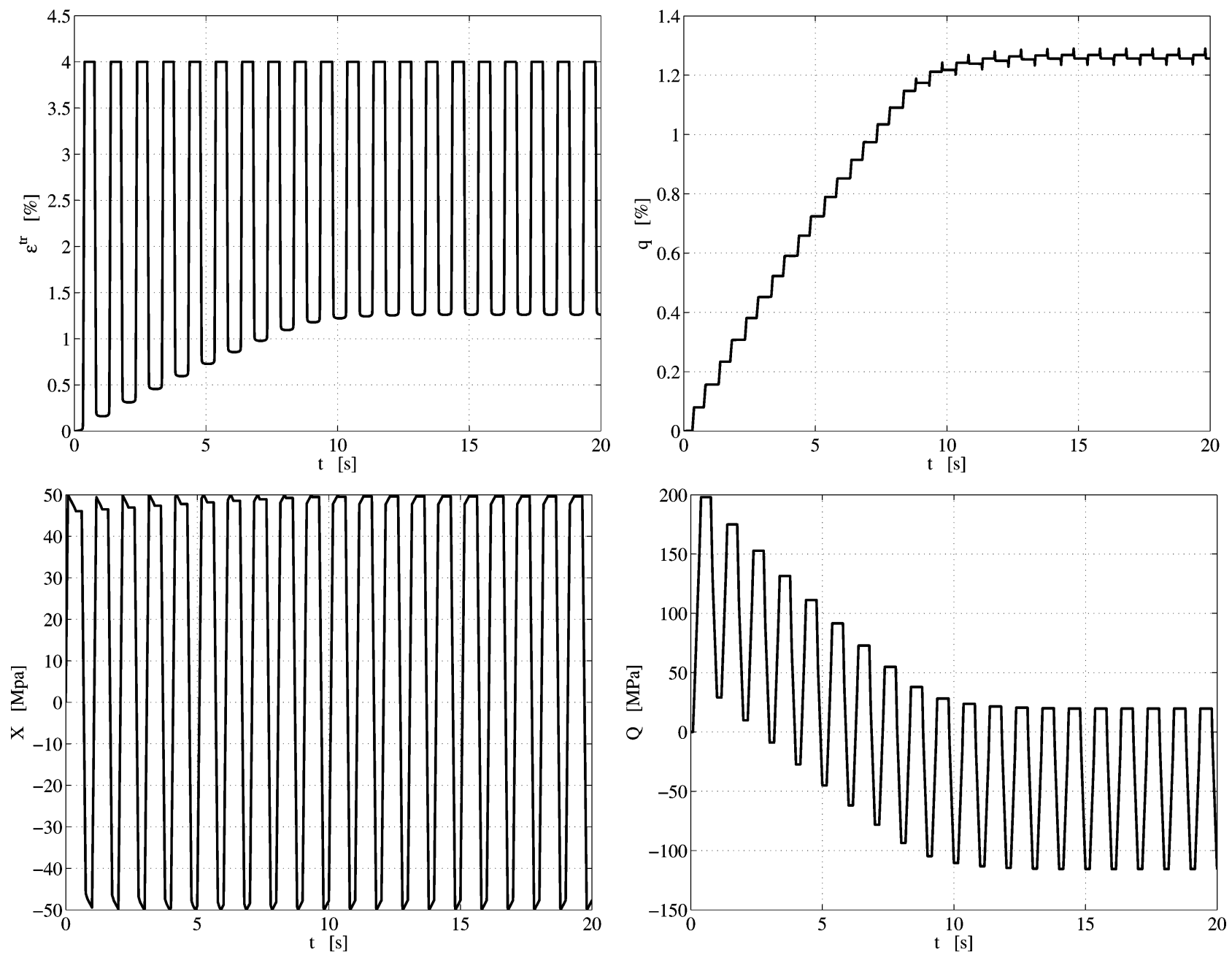

FIG. 15. Test 5: twenty tension cycles with $H=1.5 \cdot 10^{4} \mathrm{MPa}, A=10^{3} \mathrm{MPa}$ and $\mathrm{\kappa}=2 \%$. Output histories for $\varepsilon^{t r}, q, X$ and $Q$.
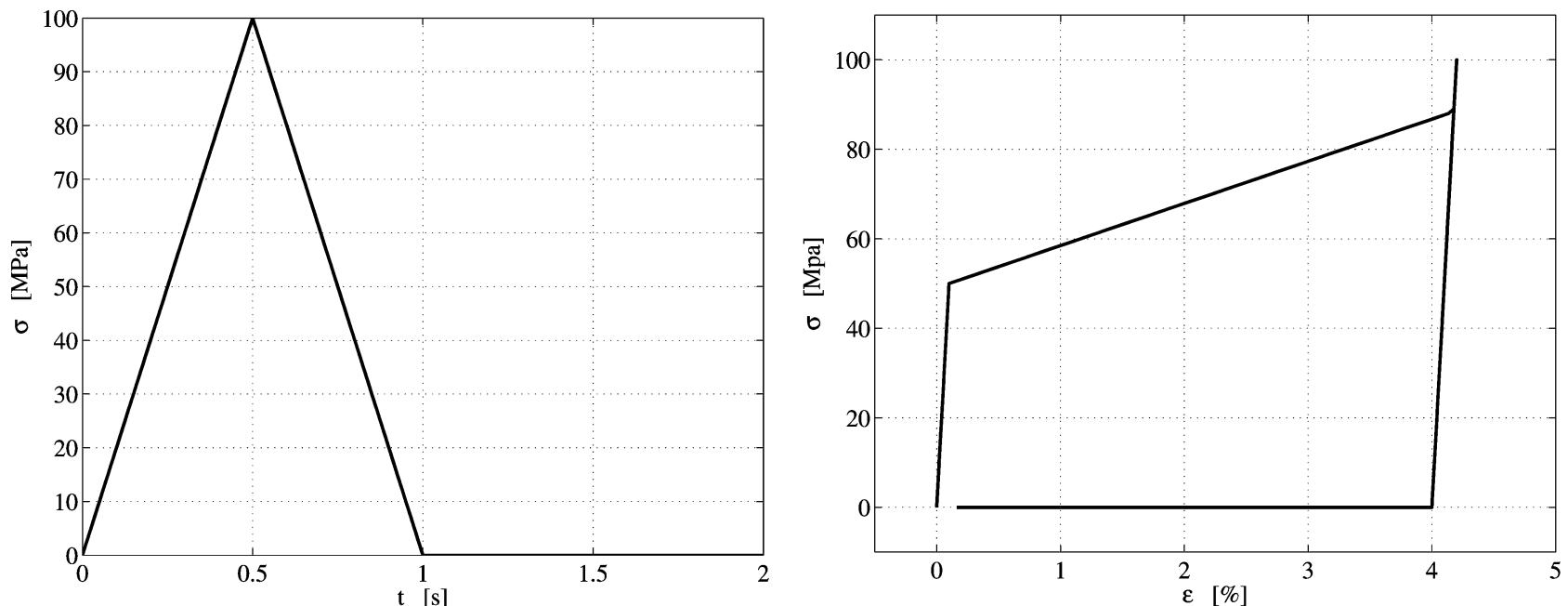

FIG. 16. Test 6: tension cycle with $T<M_{f}$ and strain recovery by heating; $H=0 \mathrm{MPa}, A=10^{3} \mathrm{MPa}$ and $\mathrm{k}=2 \%$. Input stress history and stress-strain output. 

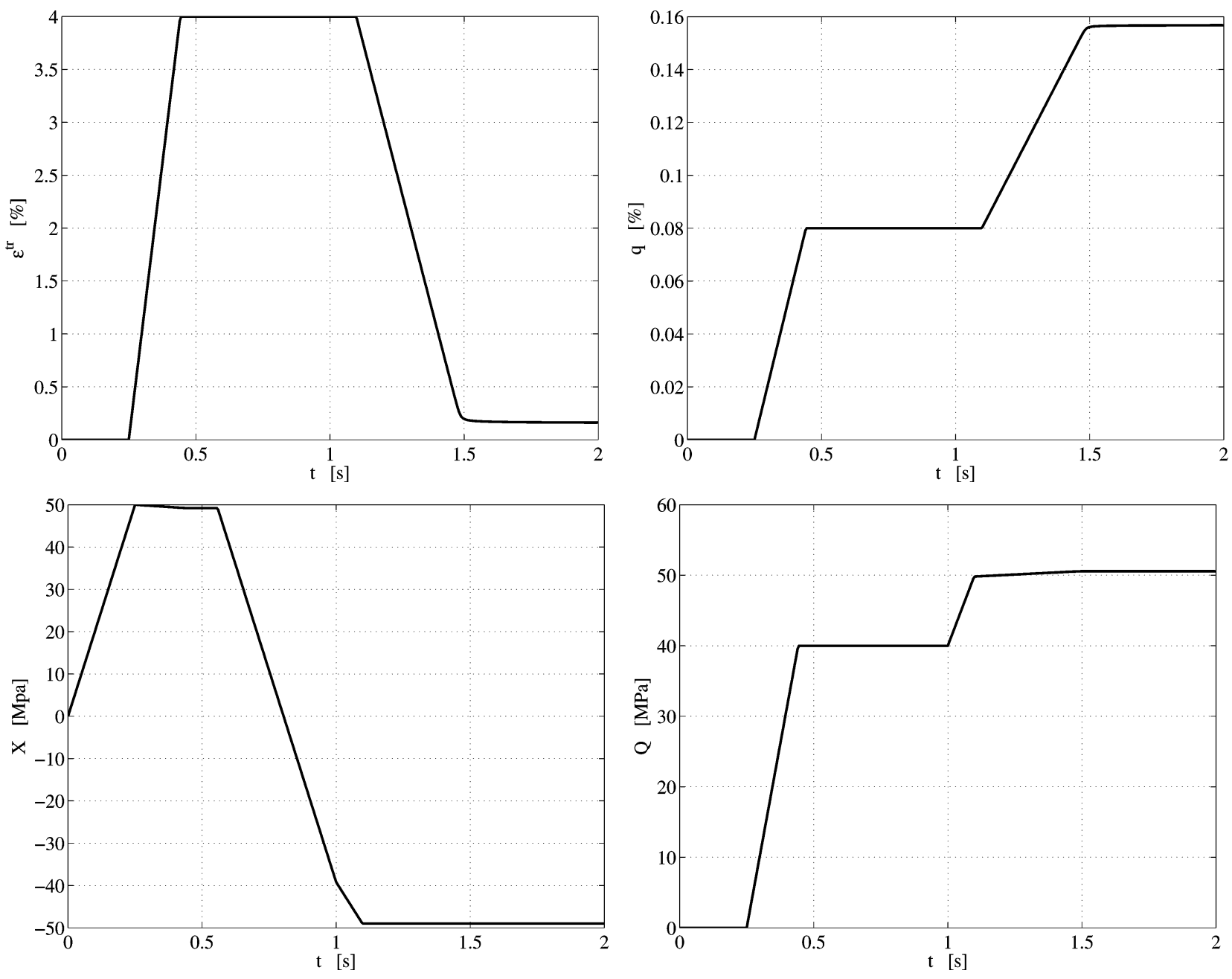

FIG. 17. Test 6: tension cycle with $T<M_{f}$ and strain recovery by heating; $H=0 \mathrm{MPa}, A=10^{3} \mathrm{MPa}$ and $\mathrm{\kappa}=2 \%$. Output histories for $\varepsilon^{t r}, q, X$ and $Q$.

\section{CONCLUSIONS}

In this paper, we have proposed a new $1 \mathrm{D}$ constitutive model for describing the macroscopic behaviour of SMA. As compared with the model proposed in $[13,14]$ and taken as a basis for the development of this new one, it is capable of including also permanent inelasticity, which can be described both with a saturating or a non-saturating evolution; degradation effects can be included as well. The good behaviour of the model has been proved by means of a number of numerical tests.

The development of a 3D version of the presented model and of a detailed algorithmic strategy for its solution will be the subject of future communications.

\section{ACKNOWLEDGEMENTS}

The authors would like to thank Doctor Ulisse Stefanelli (Istituto di Matematica Applicata e Tecnologie Informatiche del CNR, Pavia) for the fruitful discussions on the mathematical framework of the model. This work has been partially developed within the joint French-Italian "Lagrange laboratory" project and has been partially supported by the Ministero dell'Istruzione, dell'Università e della Ricerca (MIUR) through the research program "Shape-memory alloys: constitutive modeling, structural analysis and design for innovative biomedical applications," as well as by the European Project HPRN-CT2002-00284 "New Materials, Adaptive Systems and their Nonlinearities. Modelling, Control and Numerical Simulation.”

\section{REFERENCES}

1. Duerig, T. W., Melton, K. N., Stökel, D., and Wayman, C. M., editors, Engineering aspects of shape memory alloys, Butterworth-Heinemann, 1990.

2. Duerig, T. W., and Pelton, A. R., editors, SMST-2003 Proceedings of the International Conference on Shape Memory and Superelastic Technology Conference, ASM International, 2003.

3. Abeyaratne, R., and Kim, S. J., "Cyclic effects in shape-memory alloys: A one-dimensional continuum model,' International Journal of Solids and Structures 34, 3273-3289 (1997). 
4. Auricchio, F., and Lubliner, J., "A uniaxial model for shape-memory alloys," International Journal of Solids and Structures 34, 3601-3618 (1997).

5. Bo, Z., and Lagoudas, D. C., "Thermomechanical modeling of polycrystalline SMAs under cyclic loading. Part I: Theoretical derivations," International Journal of Engineering Science 37, 1089-1140 (1999).

6. Bouvet, C., Calloch, S., and Lexcellent, C. "A phenomenological model for pseudoelasticity of shape memory alloys under multiaxial proportional and nonproportional loadings," European Journal of Mechanics A/Solids 23, 37-61 (2004).

7. Govindjee, S., and Miehe, C., "A multi-variant martensitic phase transformation model: formulation and numerical implementation," Computer Methods in Applied Mechanics and Engineering 191, 215-238 (2001).

8. Helm D., and Haupt, P., "Shape memory behaviour: modelling within continuum thermomechanics," International Journal of Solids and Structures 40, 827-849 (2003).

9. Lagoudas, D. C., and Entchev, P., "Modeling of transformation-induced plasticity and its effect on the behavior of porous shape memory alloys. Part I: constitutive model for fully dense SMAs," Mechanics of Materials 36, 865-892 (2004).
10. Levitas, V. I., "Thermomechanical theory of martensitic phase transformations in inelastic materials," International Journal of Solids and Structures 35, 889-940 (1998).

11. Peultier, B. Benzineb, T., Patoor, E., "Modelling of the martensitic phase transformation for finite element computation," Journal de Physique IV France 115, 351-359 (2004).

12. Raniecki, B., and Lexcellent, C., " $R_{L}$ models of pseudoelasticity and their specification for some shape-memory solids," European Journal of Mechanics, A: Solids 13, 21-50 (1994).

13. Souza, A. C., Mamiya, E. N., and Zouain, N., "Three-dimensional model for solids undergoing stress-induced phase transformations," European Journal of Mechanics, A: Solids 17, 789-806 (1998).

14. Auricchio, F., and Petrini, L., "A three-dimensional model describing stresstemperature induced solid phase transformations. Part I: solution algorithm and boundary value problems," International Journal for Numerical Methods in Engineering 61, 807-836 (2004).

15. Arrigoni, M., Auricchio, F., Cacciafesta, V., Petrini, L., and Pietrabissa, R., "Mechanical characterization of orthodontic superelastic Ni-Ti wires," Journal de Physique IV France 11, 577-582 (2001).

16. Simo, J. C., and Hughes, T. J. R., Computational Inelasticity, SpringerVerlag, New York, 1998. 